\section{Spatholobus Suberectus \\ Column Extract Suppresses Dendritic Cell Maturation and has Therapeutic Potential for Psoriasis}

\author{
Yan Wang ${ }^{1,2}$, Jingxia Zhao ${ }^{1,2}$, Tingting $\mathrm{Di}^{1,2}$, Xiaolong $\mathrm{Xu}^{1,2}$, \\ Mingxing Wang ${ }^{1,2}$, Yujiao Meng ${ }^{1,2}$, Xiangjiang $\mathrm{Xie}^{1,2}$, Zhitong \\ Ruan $^{1,2}$, Lu Zhang ${ }^{1,2}$, Yan Lin ${ }^{1,2}$, Xin Liu ${ }^{1,2}$, Ning Wang ${ }^{1,2}$ and \\ Ping $\mathbf{L i}^{1,2 *}$ \\ ${ }^{1}$ Beijing Key Laboratory of Clinic and Basic Research with Traditional \\ Chinese Medicine on Psoriasis, Beijing Hospital of Traditional Chinese \\ Medicine, Affiliated with Capital Medical University, Beijing, China \\ ${ }^{2}$ Beijing Institute of Traditional Chinese Medicine, Beijing, China
}

\begin{abstract}
Spatholobus Suberectus Column Extract (SSCE) is a natural product from the plant Spatholobus suberectus Dunn, a kind of Traditional Chinese Medicine, which is widely used to invigorate the circulation of blood and replenish blood, has antitumor and anticoagulant properties and improves hematopoiesis. This study evaluated the clinical effects of Spatholobus Suberectus Column Extract (SSCE) in an Imiquimod (IMQ) induced psoriasis mouse model and investigated its role in regulating the differentiation and maturation of Dendritic Cells (DCs). BALB/c mice were used to establish the animal model for psoriasis-like skin lesion; SSCE at $12 \mathrm{mg} / \mathrm{kg}$ (high), 6 $\mathrm{mg} / \mathrm{kg}$ (medium) and $3 \mathrm{mg} / \mathrm{kg}$ (low) respectively, were intragastrically administered. Psoriasis Area and Severity Index (PASI) was used to evaluate the skin lesions. Histological changes, the thickness of epidermis and the quantity of CD11c+ DCs in skin lesion and spleens were measured. In vitro experiments, bone marrow cells of mice were obtained, and CD11c+ cells were isolated. DCs with a mature state in differentiation and function were identified by flow cytometry. The influence of DCs on proliferation of allogenic lymphocytes was analyzed with CCK-8. SSCE treatment alleviated psoriasis-like skin with the decreased Psoriasis Area and Severity Index (PASI) score and obviously reduced the vertical thickness of epidermis. Besides, SSCE treatment decreased the quantity of CD11c+ DCs in skin lesions and spleens. Furthermore, SSCE reduced R848-induced murine bone marrow-derived DC maturation, characterized by reduced expression of CD80/86 and inhibited the alloproliferation of T cells.
\end{abstract}

*Corresponding author: Ping Li, Beijing Key Laboratory of Clinic and Basic Research with Traditional Chinese Medicine on Psoriasis, Beijing Hospital of Traditional Chinese Medicine, Capital Medical University, Beijing, 100069 China; Beijing Institute of Traditional Chinese Medicine, Beijing, China, Tel: +86 01052176679; Fax: +86 02164085875 ; E-mail : Liping411@126.com

Citation: Wang Y, Zhao J, Di T, Xu X, Wang M, et al. (2017) Spatholobus Suberectus Column Extract Suppresses Dendritic Cell Maturation and has Therapeutic Potential for Psoriasis. J Altern Complement Integr Med 3: 017.

Received: November 22, 2016; Accepted: December 28, 2016; Published: January 12, 2017
SSCE inhibited DC function and had potential as a therapeutic agent for psoriasis.

Keywords: Dendritic cells; Psoriasis; Spatholobus suberectus column extract

\section{Introduction}

Psoriasis is a chronic inflammatory disorder of the skin affecting approximately $3 \%$ of the world's population. It is the most common chronic inflammatory skin disease characterized by epidermal hyperplasia, scaling and erythematous plaque formation [1]. Although the pathogenesis of psoriasis is not fully elucidated, it is widely accepted that cellular infiltration of T cells, Dendritic Cells (DCs) and neutrophils is important in the pathogenesis of psoriasis by provoking inflammation [2].

DCs are key players in the immune mechanisms surrounding psoriasis as well as other autoimmune diseases [3]. DC-induced cytokine production subsequently stimulated T-cell activity [4]. A cross-talk between DCs and T cells is thought to be responsible for the disease development [5]. Recently, the clearance of psoriasis using targeted immunotherapies demonstrates the important role of DCs in the pathogenesis of psoriasis.

Spatholobus Suberectus Column Extract (SSCE) is a natural product from the plant Suberect spatholobus Stem, a kind of Traditional Chinese Medicine, which is widely used to invigorate the circulation of blood and replenish blood, has antitumor and anticoagulant properties, and improves hematopoiesis [6-8]. Current research has shown its potential value in immunomodulation and the management of autoimmune diseases. Considering the pharmacological effects of Suberect spatholobus stem, it was speculated that these substances could block the pathological changes brought about by psoriasis in various aspects $[9,10]$.

This study determined whether SSCE exhibited antipsoriatic activity in a mouse model. It was the first study to evaluate the effects of SSCE on DCs in vitro and in vivo.

\section{Materials and Methods}

\section{Extraction and isolation of Spatholobus suberectus Dunn}

The whole plants of Spatholobus suberectus Dunn (0.1 kg) were bought from Beijing Hospital of Chinese Medicine. The dried and milled whole plants of Spatholobus suberectus Dunn $(0.1 \mathrm{~kg})$ were soaked with $500 \mathrm{~mL} 80 \%$ ethanol solution overnight, and extracted with water under reflux for thrice. The extracts were filtrated by 180 mesh sieve filtration, concentrated to thick paste then vacuum dying to get crude extract Spatholobus suberectus Dunn. The crude extract was dissolved with deionized water and centrifugated, the supernatant was placed in the polyamide column to elute and collect the, SSCE.

\section{Animals and grouping}

Male BALB/c mice (18 to $20 \mathrm{~g}$ ) were bought from Beijing Huafukang Biotechnology Stock Company Ltd (License No: SCXK (Jing) 20090007). All animal experimentations were performed in accordance with the guidelines for the care and use of Laboratory Animals, 
(Beijing Institute of Traditional Chinese Medicine). This animal study was approved by the Animal Care and Scientific Committee of Beijing Institute of Traditional Chinese Medicine. Mouse were randomized into four groups: (1) high-dose SSCE group (SSCE-H) with animals intragastrically administered SSCE dissolved in normal saline at a dose of $12 \mathrm{mg} / \mathrm{kg} /$ day for 6 days; (2) medium-dose SSCE group (SSCE-M), SSCE at $6 \mathrm{mg} / \mathrm{kg} /$ day for 6 days; (3) low-dose SSCE group (SSCE-L), SSCE at $3 \mathrm{mg} / \mathrm{kg} /$ day/for 6 days [11]; (4) Model group (Model), normal saline of the same volume as in SSCE-treatment groups for 6 days; (5) methotrexate (Shanghai Pharmaceutical Co., Ltd. Shanghai) group (MTX), with animals receiving methotrexate dissolved in normal saline at $1 \mathrm{mg} / \mathrm{kg} /$ day for 6 days. Mice treated with Vaseline (Lanlian Feitian Petrochemical Co., Ltd., Hebei) and intragastrically normal saline were used as controls (Ctrl). After 6-days continuous treatment, the mice were executed through cervical vertebra dislocation and their skin was collected. The skin was separated into two parts for Hematoxylin and Eosin (H\&E) staining after preparing paraffin sections and immunohistochemistry.

\section{Scoring of skin inflammation severity}

To score the severity of back skin inflammation, an objective scoring system was developed based on the clinical Psoriasis Area and Severity Index (PASI). Erythema, scaling and thickening were scored independently on a scale from 0 to 4 : 0 , none; 1 slight; 2 moderate; 3 marked; 4 very marked. The score for each group was averaged, and trend lines were generated to observe the changes in mouse skin lesions[12].

\section{Histology}

Tissue slices $(5 \mu \mathrm{m})$ were cut from the paraffin sections and stained with $\mathrm{H} \& \mathrm{E}$ for pathological observation by light microscopy. Epidermal thickness was accurately measured using the Image Pro Plus software (Leeds Precision Instruments, MN, USA).

\section{Immunohistochemistry}

Paraffin-embedded sections were dewaxed for $1 \mathrm{~h}$ with xylene and placed into a sodium citrate solution for $10 \mathrm{~min}$ at $95^{\circ} \mathrm{C}$ in a water bath for antigen retrieval. Then, the sections were fixed in $3 \% \mathrm{H}_{2} \mathrm{O}_{2}$ for $10 \mathrm{~min}$ at room temperature, and the slides were incubated overnight at $4^{\circ} \mathrm{C}$ with primary antibodies against CD3 (1:200, Abcam, USA), PCNA (1:800, Abcam, USA) and Ki67 (1:200, Abcam, USA). This was followed by incubation for $30 \mathrm{~min}$ with secondary antibodies. Frozen sections were fixed in $3 \% \mathrm{H}_{2} \mathrm{O}_{2}$ for $10 \mathrm{~min}$ at room temperature. Slides were incubated overnight at $4^{\circ} \mathrm{C}$ with primary antibodies against CD11c (1:200, Abcam, USA), followed by incubation for 30 min with secondary antibodies. Slides were observed with Olympus DP20 (Japan) and semi-quantification of the staining was performed independently by two researchers using IPP6.0. Each index was presented as integrated Optical Density (OD).

\section{Cell culture}

C57 mice were executed by cervical dislocation, immersed in 75\% alcohol for 2-3 min. The skin was cut off to take out and bony ends were cut off. RPMI1640 (HyClone, USA) was extracted using a syringe, and the bone marrow cells were washed. Tris- $\mathrm{NH}_{4} \mathrm{Cl}$ buffer was added to remove the red cells. Then, the cells were counted and seeded into a six-well plate with flat bottom at a density of $0.5-1 \times 10^{6} / \mathrm{mL}$ with RPMI 1640 medium containing granulocyte macrophage-colony-stimulating factor and interleukin-4 (Pepro Tech, USA), $100 \mathrm{ng} /$ $\mathrm{mL}$ each and cultured at $37^{\circ} \mathrm{C}$ with $5 \% \mathrm{CO}_{2}$. On Day 6, CD11c+ cells were purified using a magnetic bead method (Stem Cell, USA), and CD11c expression levels on the cell surface were detected by flow cytometry (BD, USA) to identify DCs. Cells were counted by Handheld Automated Cell Counter (Millipore, USA).

\section{Cell treatment}

On Day 6, the cells were divided into five groups the control group received no additional treatment, the model group was treated with 1 $\mu \mathrm{g} / \mathrm{mL} \mathrm{R} 848$ for $24 \mathrm{~h}$ and the other three groups were pretreated with SSCE at the concentrations of $15,7.5$ and $3.75 \mu \mathrm{g} / \mathrm{mL}$ for $3 \mathrm{~h}$ and then treated with $1 \mu \mathrm{g} / \mathrm{mL}$ R848 for $24 \mathrm{~h}$.

\section{Flow cytometric analysis}

Inguinal spleen's samples from each group were minced through a 70-mm mesh to obtain single cell suspensions. Fluorescence-conjugated monoclonal Antibodies (mAbs) recognizing CD11c, CD80, CD86, MHC-II and their respective isotype controls were obtained (eBioscience, USA). Cell surface staining was performed by incubating $5 \times 10^{5}$ cells in $100 \mu \mathrm{L}$ of staining buffer containing optimal concentrations of $\mathrm{mAb}$ specific for a receptor or an Ig isotype-matched control for $20 \mathrm{~min}$. After incubation, the cells were washed with PBS (Amresco, USA). Samples were analyzed using a flow cytometer (BD, USA).

\section{Mixed homogenous lymphocyte reaction}

Cells of all groups were treated with $25 \mu \mathrm{g} / \mathrm{mL}$ mitomycin C, incubated at $37^{\circ} \mathrm{C}$ for $45 \mathrm{~min}$, and washed with phosphate-buffered saline three times. Then, the cells were suspended in an RPMI 1640 medium and seeded in round-bottomed 96-well plates at the density of $1 \times 10^{4} /$ well. Homogenous lymphocytes $\left(4 \times 10^{5}\right)$ were added into each well to make up a final volume of $200 \mu \mathrm{L}$. Three replicates were performed for each group. The cells were cultured for 5 days, and then $10 \mu \mathrm{L}$ of Cell Counting Kit-8 (CCK-8, Dojindo, Japan) was added into each well for further incubation of $3 \mathrm{~h}$. The OD was measured using a microplate reader, and the average value of three wells was calculated.

\section{Statistical analysis}

All quantitative data were expressed as the mean \pm standard deviation. Comparisons between two groups were assessed using the Student's t-test, and comparisons among three or more groups were evaluated via one-way analysis of variance followed by post hoc test with SPSS software 15.0 (IL, USA). P values less than 0.05 were considered to indicate statistical significance.

\section{Results}

\section{SSCE effectively attenuated psoriatic lesions and alleviated the histopathologic changes in IMQ-induced psoriasis-like mouse model}

To value whether SSCE was beneficial in IMQ-induced psoriasis-like mouse model, mice were treated with SSCE 12, 6 and $3 \mathrm{mg} /$ kg. After 6-days treatment with drugs, psoriatic lesions characterized by erythema, thickening and scaling were observed in the IMQ group, while SSCE significantly alleviated the clinical changes in the IMQ-treated mice (Figure 1A). Moreover, the disease severity was assessed by a clinical scoring system. It was found that SSCE significantly reduced the PASI scores after 6-days treatment (Figure 1B). 
Similar to the MTX group, SSCE effectively ameliorated the histological appearance and the infiltrated lymphocytes of the psoriatic lesions compared with the IMQ control (Figure 1C). In addition, it was also found that SSCE effectively reduced the average epidermal thickness compared with the IMQ group (Figure 1D). These data showed that SSCE could effectively alleviate the histopathologic changes in IMQ-induced psoriasis-like mouse lesions.

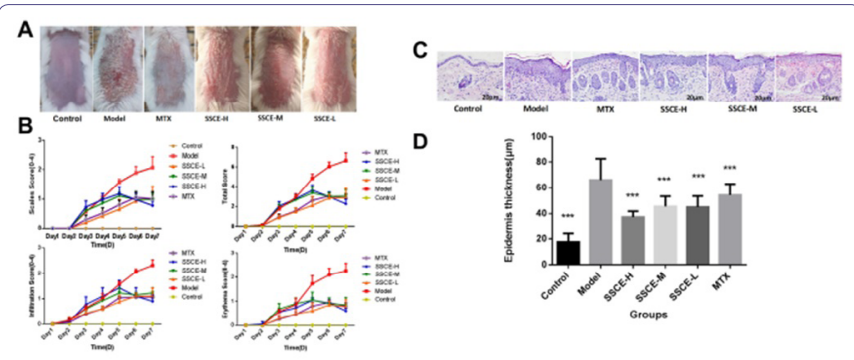

Figure 1: SSCE improved the morphological features and alleviated the histopathologic changes of IMQ-induced psoriasis in mice.

(A) Phenotypical presentation of the back skin of mice after 6 days of imiquimod treatment.

(B) Erythema, scaling and thickness of the back skin were scored daily on a scale from 0 to 4 . The cumulative score (erythema + scaling + thickness) is depicted. Symbols indicate the mean $\pm \operatorname{SD}(n=5)$.

(C) Histological changes in mice skin lesions on day 6 of imiquimod treatment and H\&E staining of the back skin of mice $(200 \times)(n=5)$.

(D) Epidermis thickness changes of skin lesions in mice $(\mu \mathrm{m}$, mean $\pm \mathrm{SD}, \mathrm{n}$ $=5$ )

${ }^{* * *} \mathrm{P}<0.001$ vs model. H\&E- Hematoxylin and eosin; IMQ- imiquimod; SDstandard deviation; SSCE- Spatholobus suberectus column extract.

\section{SSCE reduced the infiltration of $\mathrm{T}$ cells and neutrophils in IMQ-induced psoriasis-like mouse lesions}

Cellular infiltration of neutrophils, keratinocytes and T cells in lesions plays vital roles in the pathogenesis of psoriasis. Immunohistochemistry using antibody against CD3 and Ki67 showed the abundant infiltration of T cells and keratinocytes indermis in the IMQ group. However, SSCE markedly reduced the cellular infiltration of T cells and keratinocytes in dermis when compared with the IMQ group (Figure 2A-B).

\section{SSCE decreased the number of CD11c+ DCs in IMQ-in- duced psoriasis-like mouse lesions and spleens}

Immunofluorescence Assay (IFA) was used to detect the number of CD11c+DCs in the dorsal skin of IMQ-induced mice. Compared with the normal control group, the number of CD11c+DCs significantly increased in the model group but significantly reduced in the SSCE and MTX group in comparison to the model group (Figure $2 \mathrm{C}$ ). The number of CD11c+DCs in spleens was detected using flow cytometer, and the results were similar to those of skin lesion (Figure 2D).

\section{Effect of SSCE on cell viability in vitro}

Cell viability was determined by CCK-8. Bone marrow derived dendritic cells were incubated overnight in 96 -well plates with flat bottom at a density of $5 \times 10^{4}$ cells per well. After $24 \mathrm{~h}$, the cells were treated with different concentrations $(250 \mu \mathrm{g} / \mathrm{ml}, 125 \mu \mathrm{g} / \mathrm{ml}, 62.5 \mu \mathrm{g} / \mathrm{ml}, 31.25$ $\mu \mathrm{g} / \mathrm{ml}, 15 \mu \mathrm{g} / \mathrm{ml}$ and $7.5 \mu \mathrm{g} / \mathrm{ml}$ ) of SSCE for another $20 \mathrm{~h}$. Then $10 \mu \mathrm{L}$ of CCK-8 (5\%) was added into each well for further incubation of $4 \mathrm{~h}$ at $37^{\circ} \mathrm{C}$, and the $\mathrm{OD}$ was measured at $570 \mathrm{~nm}$ using a microplate reader (Bio-Rad, USA) (Figure 3).

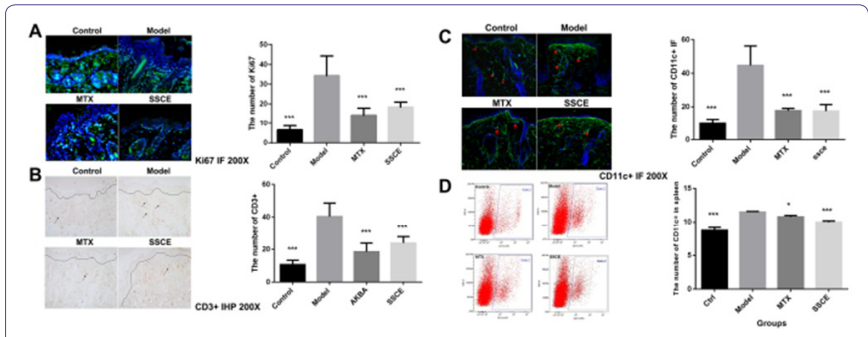

Figure 2: SSCE alleviated inflammatory cell infiltration in psoriasis-like lesions and spleens.

(A) Ki67 expression in mice lesions on day $6(n=4)$

(B) CD3 expression in mice lesions on day $6(n=4)$.

(C) $C D 11 c+$ expression in mice lesions on day $6(n=4)$.

(D) CD11c+ expression in mice spleens on day $6(n=4)$.

SSCE: Mice were treated with $12 \mathrm{mg} /\left(\mathrm{kg} \cdot\right.$ day). ${ }^{*} \mathrm{P}<0.05$ vs model, ${ }^{* *} \mathrm{P}<$ 0.001 vs model. DC- dendritic cell.

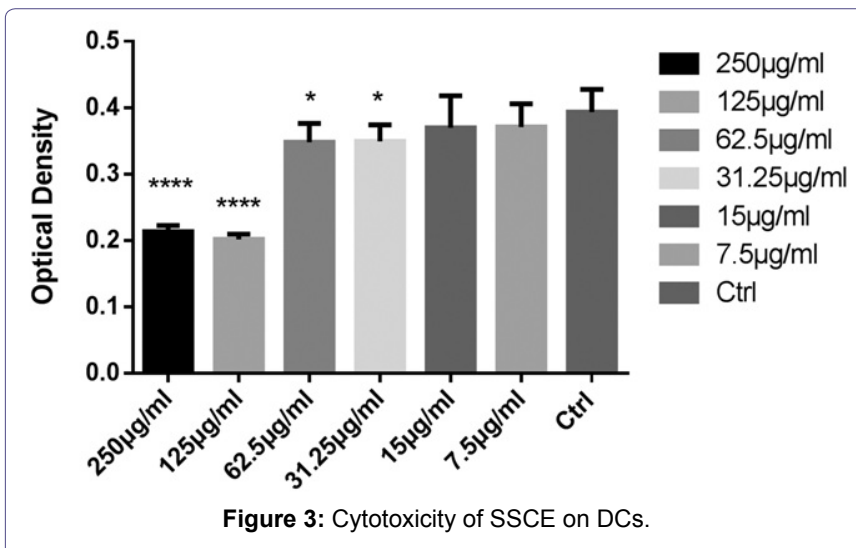

Cells were incubated with different concentrations of SSCE for 24h. Data represent the mean $\pm S D$. ${ }^{*} P<0.05,{ }^{* * * *} P<0.0001$ indicate significant differences compared with the control group. CCK-8- Cell counting kit-8; DC- dendritic cell; SD- standard deviation; SSCE- Spatholobus suberectus column extract.

\section{Effects of SSCE on the phenotypic and functional matura-} tion of DCs

As shown in figure $4 \mathrm{~A}$ and $4 \mathrm{~B}$, a phenotypic analysis showed that $95 \%$ of the cells expressed CD11c after purifying with magnetic bead method. R848 was used to induce maturation of DCs with a resultant enhanced surface expression of CD80 and CD86. SSCE treatment inhibited the R848 effect on the maturity markers in CD80 and CD86. These results indicated that SSCE inhibited DC maturation.

A main characteristic of mature DCs is their capacity of inducing allogeneic T-cell proliferation. To further investigate the effect of SSCE on the maturation of DCs, the allogeneic mixed lymphocyte reaction was examined. It was found that R848 increased CD4+ T-cell proliferation, while SSCE inhibited the capacity of allogeneic T-cell proliferation (Figure 4C).

\section{Discussion}

This study demonstrated that SSCE alleviated psoriasis-like skin with the decreased PASI score and obviously reduced the vertical thickness of epidermis. Furthermore, SSCE prevented the maturation of murine Bone Marrow Dendritic Cells (BMDCs), characterized by reduced levels of CD80/86, and reduced all oproliferation of $\mathrm{T}$ cells. These results suggested that SSCE regulated DC maturation, and that it might serve as a novel therapeutic agent for psoriasis. 


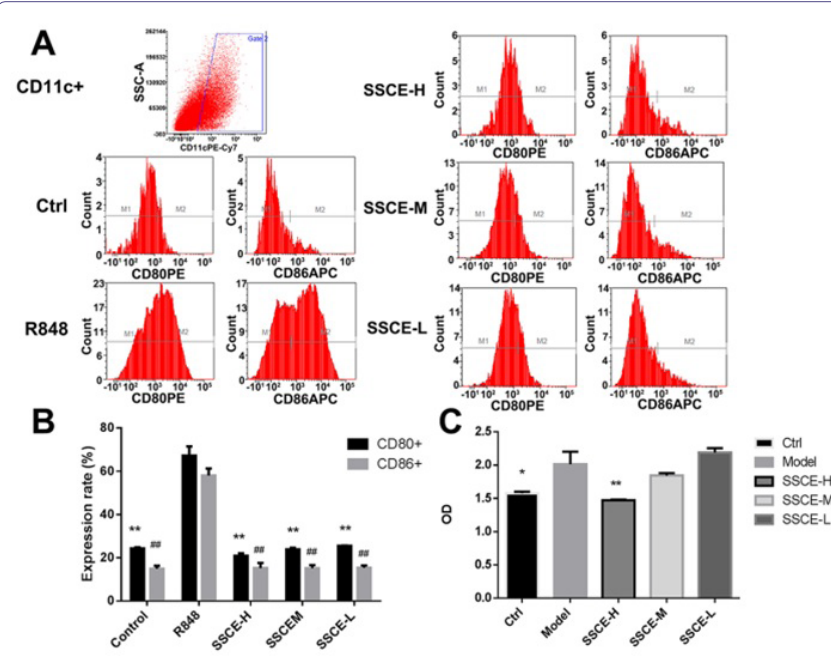

Figure 4: Effect of SSCE on maturation of BMDCs.

(A and B) Effect of SSCE on the expression of DC maturity marker.

(C) After treatment with $10 \mathrm{ng} / \mathrm{mL}$ R848 (model) or $10 \mathrm{ng} / \mathrm{mL}$ R848+ SSCE $\left(15,7.5\right.$ and $3.75 \mu \mathrm{g} / \mathrm{mL}$ ) for $24 \mathrm{~h}, 4 \times 10^{5}$ allograft lymphocytes were co-cultured with DCs of each group, and the proliferation rate of lymphocytes was measured using the CCK-8 kit. ${ }^{*} P<0.05$ and ${ }^{* *} \mathrm{P}<0.01$ vs $\mathrm{R} 848$ group. BMDCBone marrow dendritic cell; CCK-8- cell counting Kit-8; GM-CSF- granulocyte macrophage-colony-stimulating factor; IL-4- interleukin-4; SSCE- Spatholobus suberectus column extract. APC- Allophycocyanin; PE- Phycoerythrin.

Although SSCE has been reported to be an anticancer agent $[13,14]$, the effect of SSCE in psoriasis and DCs has not yet been determined. This study found that SSCE treatment alleviated IMQ-induced psoriasis mice model, inhibited BMDC maturation and suppressed proliferation of allogeneic T cells. These results suggested that SSCE could inhibit the maturation and activation of DCs and might be of potential value in treating some autoimmune diseases.

Imiquimod (IMQ) is a TLR7/8 agonist, and IMQ-induced mouse model is a classic model of psoriasis [15]. To investigate the effect of SSCE on psoriasis, the effect of SSCE on IMQ-induced psoriasis mice model was evaluated. It was observed that SSCE effectively attenuated psoriatic lesions, alleviated the histopathologic changes, reduced the infiltration of $\mathrm{T}$ cells and neutrophils in psoriasis-like mouse lesions, and reduced the number of CD11c+ DCs in mouse lesions and spleens.

DCs are thought to be the initiators of some autoimmune diseases such as psoriasis. Maturation and activation of DCs are key steps in triggering the priming of autoreactive peripheral T cells, which then drive the development of inflammatory responses [16-19]. The effect of SSCE on DCs maturation was evaluated in R848-induced BMDCs. It was observed that SSCE treatment in R848-induced BMDCs resulted in a significant reduction in the expression of DC maturity markers and inhibited the capacity of allogeneic T-cell proliferation.

\section{Conclusion}

In summary, the present study showed that SSCE inhibited DCs maturation by reducing the expression of maturity markers, suppressed the ability of allogeneic T-cell proliferation, and effectively improved the disease condition of mice with psoriasis. The present data not only clarified a new cellular mechanism for the anti-inflammatory and immunosuppressive effects of SSCE, but also indicated the therapeutic potential for psoriasis.

\section{Disclosures}

The authors have no financial conflicts of interest.

\section{Acknowledgment}

The study was supported by National Natural Science Foundation of China (No. 81403410).

\section{References}

1. Boehncke WH (2015) Etiology and Pathogenesis of Psoriasis. Rheum Dis Clin North Am 41: 665-675.

2. Rahmani F, Rezaei N (2016) Therapeutic targeting of Toll-like receptors: a review of Toll-like receptors and their signaling pathways in psoriasis. Expert Rev Clin Immunol 12: 1289-1298.

3. Liu J, Cao X (2015) Regulatory dendritic cells in autoimmunity: A comprehensive review. J Autoimmun 63: 1-12.

4. Gordon JR, Ma Y, Churchman L, Gordon SA, Dawicki W (2014) Regulatory dendritic cells for immunotherapy in immunologic diseases. Front Immunol 5: 7 .

5. Cao LY, Chung JS, Teshima T, Feigenbaum L, Cruz PD, et al. (2016) Myeloid-Derived Suppressor Cells in Psoriasis Are an Expanded Population Exhibiting Diverse T-Cell-Suppressor Mechanisms. J Invest Dermatol 136: 1801-1810.

6. Yan L, Ping L, Yan W, Xin-ran X, Lei Z, et al. (2012) Effect of Active Part of Spatholobus suberectus Flavonoid on Oxidative Stress of Human Pulmonary Adenocarcinoma Cell A549. Chinese Journal of Experimental Traditional Medical Formulae 4: 190-193.

7. Guo-wang Y, Shan G, Qi F, Ping L, Xiao-qin L, et al. (2012) Molecular mechanism of cell cycle regulation of Spatholobus suberectus flavonoids effective part on the A549 human lung adenocarcinoma cell based on CKI. China Journal of Traditional Chinese Medicine and Pharmacy 3217-3220.

8. Yan W, Ping L, Yan L, Jing-xia Z, Xin L, et al. (2011) Effect of Spatholobus suberectus column extract on apoptosis and mitochondrial transmembrane potential of non-small cell lung cancer A549 in vitro. Journal of Capital Medical University 32: 811-815.

9. J n m college (1997) Chinese medicine dictionary. Shanghai: Shanghai science and technology press, Shanghai, China.

10. Bo Y, Zhi-yu L, Qi-dong Y (2008) The development in the research of anti-tumor mechanism of flavonoids. Progress in Pharmaceutical Sciences 32 : 391-397.

11. Fu Q, Tang Y, Luo X, Yang G, He W, et al. (2009) [Anti-tumor activity and mechanism with SSCE of Spatholobus suberctus]. Zhongguo Zhong Yao Za Zhi 34: 1570-1573.

12. van der Fits L, Mourits S, Voerman JS, Kant M, Boon L, et al., (2009) imiquimod-induced psoriasis-like skin inflammation in mice is mediated via the IL-23/ IL-17 Axis. J Immunol 182: 5836-5845.

13. Shan G, Guo-wang Y, Qi F, Ping L, Xiao-qin L, et al. (2010) Molecular mechanism of cell cycle regulation of Spatholobus suberectus flavonoids Effective part (SSCE) on the A549 human lung adenocarcinoma cell. Chinese Journal of Basic Medicine in Traditional Chinese Medicine 247-250.

14. Wang G-C (1999) The family of CIP-KIP, Foreign Medical Sciences. Section of Molecular Biology. 31: 17-21.

15. Singh M, Khong H, Dai Z, Huang XF, Wargo JA, et al. (2014) Effective innate and adaptive antimelanoma immunity through localized TLR7/8 activation. J Immunol 193: 4722-4731.

16. Saha C, Das M, Stephen-Victor E, Friboulet A, Bayry J, et al. (2016) Differential Effects of Viscum album Preparations on the Maturation and Activation of Human Dendritic Cells and CD4 ${ }^{+}$T Cell Responses. Molecules 21: 912.

17. Said A, Weindl G (2015) Regulation of Dendritic Cell Function in Inflammation. J Immunol Res 2015: 743169. 
Citation: Wang Y, Zhao J, Di T, Xu X, Wang M, et al. (2017) Spatholobus Suberectus Column Extract Suppresses Dendritic Cell Maturation and has Therapeutic Potential for Psoriasis. J Altern Complement Integr Med 3: 017.

- Page 5 of $5 \cdot$

18. Kim TG, Kim DS, Kim HP, Lee MG (2014) The pathophysiological role of dendritic cell subsets in psoriasis. BMB Rep 47: 60-68.
19. Batal I, Azzi J, Mounayar M, Abdoli R, Moore R, et al. (2014) The mechanisms of up-regulation of dendritic cell activity by oxidative stress. J Leukoc Biol 96: 283-293. 